\title{
BMJ Open Prospective registration trends, reasons for retrospective registration and mechanisms to increase prospective registration compliance: descriptive analysis and survey
}

To cite: Hunter KE, Seidler AL, Askie LM. Prospective registration trends, reasons for retrospective registration and mechanisms to increase prospective registration compliance: descriptive analysis and survey. BMJ Open 2018;8:e019983. doi:10.1136/ bmjopen-2017-019983

- Prepublication history and additional material for this paper are available online. To view these files, please visit the journal online (http://dx.doi. org/10.1136/bmjopen-2017019983).

Received 6 October 2017 Revised 12 January 2018 Accepted 6 February 2018

Check for updates

NHMRC Clinical Trials Centre, University of Sydney, Camperdown, New South Wales, Australia

Correspondence to

Kylie Elizabeth Hunter;

kylie.hunter@ctc.usyd.edu.au

\section{ABSTRACT}

Objectives To analyse prospective versus retrospective trial registration trends on the Australian New Zealand Clinical Trials Registry (ANZCTR) and to evaluate the reasons for non-compliance with prospective registration.

Design Part 1: Descriptive analysis of trial registration trends from 2006 to 2015. Part 2: Online registrant survey.

Participants Part 1: All interventional trials registered on ANZCTR from 2006 to 2015. Part 2: Random sample of those who had retrospectively registered a trial on ANZCTR between 2010 and 2015.

Main outcome measures Part 1: Proportion of prospective versus retrospective clinical trial registrations (ie, registration before versus after enrolment of the first participant) on the ANZCTR overall and by various key metrics, such as sponsor, funder, recruitment country and sample size. Part 2: Reasons for non-compliance with prospective registration and perceived usefulness of various proposed mechanisms to improve prospective registration compliance.

Results Part 1: Analysis of the complete dataset of 9450 trials revealed that compliance with prospective registration increased from $48 \%$ (216 out of 446 trials) in 2006 to $63 \%(723 / 1148)$ in 2012 and has since plateaued at around $64 \%$. Patterns of compliance were relatively consistent across sponsor and funder types (industry vs non-industry), type of intervention (drug vs non-drug) and size of trial $(n<100,100-500,>500)$. However, primary sponsors from Australia/New Zealand were almost twice as likely to register prospectively (62\%; 4613/7452) compared with sponsors from other countries with a WHO Network Registry (35\%; 377/1084) or sponsors from countries without a WHO Registry (29\%; 230/781). Part 2: The majority $(56 \% ; 84 / 149)$ of survey respondents cited lack of awareness as a reason for not registering their study prospectively. Seventy-four per cent (111/149) stated that linking registration to ethics approval would facilitate prospective registration.

Conclusions Despite some progress, compliance with prospective registration remains suboptimal. Linking registration to ethics approval was the favoured strategy among those sampled for improving compliance.
Strengths and limitations of this study

- To describe trends in prospective registration, this study used the complete dataset of 9450 trials registered on Australian New Zealand Clinical Trials Registry (ANZCTR) from 2006 to 2015

- To our knowledge, this is the first survey of its kind to obtain feedback directly from registrants about reasons for retrospective registration and mechanisms to address this.

- A limitation is that we only have data for trials that were registered on the ANZCTR. Unregistered trials are not taken into account in this analysis, and therefore, overall compliance with prospective registration is lower than reported.

\section{INTRODUCTION}

Prospective trial registration is the process whereby key details about a planned clinical trial are made publicly available on a recognised clinical trial registry before enrolment of the first participant. This reduces the temptation to either not publish or only publish selective results from completed trials. Thus, prospective trial registration is now widely recognised as a key strategy to increase research transparency and accountability by minimising publication bias and selective outcome reporting bias. ${ }^{1-5}$ It has been described by medical journal editors as 'the single most valuable tool we have to ensure unbiased reporting'. 6

An increasing number of clinical trials registries have been established throughout the world ${ }^{7}$ and prospective trial registration now has the support of numerous organisations nationally and internationally. The International Committee of Medical Journals Editors (ICMJE), which includes many of the world's leading journals, declared in 2004 that they would not consider a trial for 
publication without evidence that it had been registered in a publicly accessible trial registry prior to enrolment of the first participant. ${ }^{8}$ The Declaration of Helsinki now explicitly states that 'every clinical trial must be registered in a publicly accessible database before recruitment of the first subject'. ${ }^{9}$ The Declaration is the cornerstone document guiding the ethical conduct of research in humans. The WHO considers the registration of all interventional trials to be 'a scientific, ethical and moral responsibility'. ${ }^{10}$

However, 10 years after the ICMJE declaration, almost $30 \%$ of trials published in high-impact journals were still not registered prospectively (and thus did not comply with the policy). ${ }^{11}$ By not registering details about a trial before enrolment of the first participant, the main tenant of research transparency is undermined. Some trial registries (including the Sri Lanka Clinical Trials Registry and from 2018 the Clinical Trials Registry-India) have sought to deal with this by not permitting the registration of trials after enrolment of the first participant, that is, only permitting prospectively registered trials to be included on their registries. The Australian New Zealand Clinical Trials Registry (ANZCTR) has adopted a different approach, by still allowing registration after enrolment of the first participant (ie, retrospective registration). This is to encourage the registration of (and thus public access to) as many trials as possible. However, in an effort to encourage prospective registration in January 2013 the ANZCTR began publicly labelling records as being either prospectively or retrospectively registered on their website. It was hoped that this would make it easier for journal editors and other regulatory bodies to enforce sanctions for non-compliance with the prospective registration obligation.

While there is international consensus on the importance of prospective registration, many researchers do not comply with this obligation, and there is little information on potential reasons for this. To improve prospective registration, more information on rates and trends in retrospective registration is required, and the types of trials that are more likely to be retrospectively registered need to be identified. Furthermore, information is needed on the reasons trialists do not comply with the requirement and how the process could be improved to reach higher compliance rates.

The overarching aim of this study was to describe prospective versus retrospective trial registration trends on the ANZCTR and to evaluate reasons for non-compliance with prospective registration (registration before enrolment of first participant).

\section{METHODS}

\section{Key objectives}

1. To describe the proportion of prospective versus retrospective clinical trial registrations on the ANZCTR from 2006 to 2015 and determine any impact of introducing the prospective/retrospective registration label in January 2013.
2. To analyse prospective registration compliance on the ANZCTR by key trial characteristics.

3. To determine median time from registration on the ANZCTR to trial start date by year and key trial characteristics.

4. To determine reasons for non-compliance with the prospective registration requirement.

5. To evaluate the perceived usefulness of various proposed mechanisms to improve prospective registration compliance.

\section{Part 1: Retrospective descriptive analysis of the cohort of registered trials on ANZCTR \\ Included studies}

Part 1 of the study was a descriptive analysis of prospective registration trends using data from all interventional studies registered on the ANZCTR from 1 January 2006 until 31 December 2015, regardless of the condition of interest. Although the ANZCTR was established in 2005 , data from this year were excluded from the analysis because: (1) the registry was launched in July 2005 and thus data for the full year are not available and (2) after the ICMJE introduced their prospective registration requirement in July 2005, they allowed authors a grace period of a few months for registration of ongoing trials, ${ }^{8}$ thus a large proportion of studies were registered retrospectively in 2005. Although the ANZCTR accepts observational studies for registration, they were excluded from this analysis as the ICMJE's statement applies only to clinical trials evaluating health-related interventions. ${ }^{8}$ Interventional studies were extracted based on the 'Study type' field on the ANZCTR registration form, which requires registrants to select either 'interventional' or 'observational'.

\section{Measures}

As per the WHO International Clinical Trials Registry Platform (ICTRP) International Standards for Clinical Trial Registries, ${ }^{12}$ we defined studies as prospectively registered if their registration date (the date their registry entry was approved, not the date it was submitted) occurred prior to the actual date of first participant enrolment listed by the registrant. Where the actual date was not available, we used the anticipated date of first participant enrolment.

In 2013, a publicly viewable prospective/retrospective label was added to all registered records. This label is automatically generated by the ANZCTR system based on the registration date and the date of first participant enrolment provided by the registrant, as described above. If the registration date occurs after the listed start date, the record was labelled as retrospectively registered. If it occurs prior to the start date, it was labelled as prospectively registered.

We analysed prospective registration trends by the following study characteristics, which were recorded by the registrant at the time of registration or update:

- Primary sponsor type: the individual, organisation, group or other legal person taking on responsibility 
for securing the arrangements to initiate and/or manage a study, usually the principal investigator (eg, commercial sector, government body, university, individual);

- Funding type: sources of monetary or material or infrastructure support for the study (eg, commercial sector, government body, university, individual);

- Registrant country, primary sponsor country, funding country, recruitment country: country was categorised into Australia/New Zealand versus countries with a registry in the WHO Registry Network (here on referred to as WHO Registry) versus countries without a WHO Registry. This categorisation was due to registration processing triaging: while the ANZCTR accepts entries from all countries, entries from Australia/New Zealand are prioritised, and countries with a WHO Registry are encouraged to register with their local registry;

- Recruitment country was further categorised into national versus multinational recruitment: recruitment in only one country versus recruitment in two or more countries;

- Intervention type, for example, treatment: drugs, diagnosis/prognosis, early detection/screening, prevention, treatment: surgery, treatment: devices, treatment: other, rehabilitation, lifestyle, behaviour, other interventions.

- Target sample size: The raw number provided by the registrant was categorised as $\mathrm{n}<100$ versus $\mathrm{n}=100$ 500 versus $n>500$;

- Study phase: Phases of investigation, usually applied to drug trials, ranging from phase 0 (includes exploratory, first-in-human trials) to phase 4 (postmarketing study to delineate additional information). The ANZCTR allows combination options (eg, phase 2/3), but for the purpose of this analysis combined phases were coded according to the lowest included phase (eg, phase 2/3 was coded to phase 2). This is an optional field on the ANZCTR registration form;

- Health condition: up to three per study, coded into Australian National Health Priority Area (NHPA) conditions ${ }^{13}$ including arthritis and musculoskeletal conditions, asthma, cancer, cardiovascular disease, dementia, diabetes, injury, mental health and obesity, if applicable.

The trial registration date appears on all registered records and is the date when final approval was given and the registration ID (ANZCTR trial registration number (ACTRN)) was issued. Trial start date was obtained from the 'Actual date of first participant enrolment' field. If this field was incomplete then the 'Anticipated date of first participant enrolment' field was used.

For definitions of each of the ANZCTR data fields collected, please refer to the ANZCTR Data Item Definitions document available at www.anzctr.org.au/docs/ ANZCTR Data field explanation.pdf
Analyses

The proportion of prospectively and retrospectively registered trials was calculated and plotted for all trials each year from 2006 to 2015. Prospective registration rates per year were compared before and after the introduction of the prospective registration label in 2013. To examine prospective registration compliance by key study characteristics, prospective versus retrospective registration rates were compared by funding type, intervention type, target sample size, national versus multinational, phase, NHPA health condition and recruitment country. Median time between trial registration date and trial start date was calculated for all included studies, and by registrant country, primary sponsor country, funding country and recruitment country. Analyses were descriptive and were conducted using Microsoft Excel and Stata IC V.14. No inferential statistical analyses were employed since our descriptive analysis included the entire dataset from all interventional trials registered on ANZCTR, which is our population of interest. The observed differences are the real population differences, and no inferential statistical analyses are required to test their generalisability. Any missing data were excluded from analyses.

\section{Part 2: Online registrant survey}

Included studies

Part 2 of the study was a cross-sectional online survey conducted in April-May 2017 sampling a random $15 \%$ of researchers who had retrospectively registered their interventional studies on ANZCTR between 1 January 2010 and 31 December 2015, stratified by registration year, primary sponsor type and registrant country. With about 2970 interventional studies registered retrospectively in this predefined 6-year period, some registrants registering multiple studies and an expected response rate of around $25 \%$, a $15 \%$ sample $(n=445)$ was required to reach the target sample size of $n=100$. Stratification variables were chosen to obtain a representative sample and therefore minimise bias. We chose not to include records registered prior to January 2010 as it was thought to be unreasonable to expect a registrant to recall reasons for retrospective registration greater than 7 years ago. The survey was conducted using the SurveyMonkey software (www.surveymonkey. com), and eligible registrants were invited to participate via e-mail, using the e-mail address provided for the purposes of registration.

\section{Measures}

The survey consisted of three categorical questions asking about:

- The registrant's role (eg, principal investigator, trial coordinator, research student);

- Reasons for non-compliance with prospective registration (eg, lack of awareness, lack of organisation);

- How retrospective registration may have been prevented (eg, linking registration to ethics). 
Respondents could select as many response options as applicable and could also add free text notes if desired. The list of response options was carefully determined by two experts in the field (both with over 8 years of experience at ANZCTR) based on the feedback they received from registrants and information from a recently published editorial on trial registration in the $B M J{ }^{6}{ }^{6}$ The full survey, including all available response options, can be found in online supplementary appendix 1.

Respondents could choose to remain anonymous or provide their name and/or ACTRN. Where either of these identifiers were provided, additional metrics (such as registrant country, approval date, sponsor type, etc) were extracted from the full record for further analysis. Analyses

Categorical survey responses were combined into broader categories for presentation of results. Where possible, free text responses were manually coded into the appropriate category; for responses that did not fit into an existing category new categories were created. The proportion of participants selecting each response category was calculated and presented. For incomplete surveys, all available responses were used. Data from SurveyMonkey was exported to Microsoft Excel and the open-source software $\mathrm{R}^{14}$ for data cleaning, coding and analysis.

For both parts of the study, all objectives, measures and analyses were specified in a protocol prior to data extraction and analyses. The survey was approved by the University of Sydney Human Research Ethics Committee (Project no. 2016/805) and completion of the survey was deemed to constitute informed consent.
RESULTS

Part 1: Retrospective descriptive analysis of the cohort of registered trials in ANZCTR

A total of 9450 trials were registered on the ANZCTR between 2006 and 2015 and are included in part 1 of the study. The types of interventions administered in these trials are shown in online supplementary appendix 2 , and the distribution of registrant country is shown in online supplementary appendix 3 . Data collected for trials registered in 2006-2007 were not as complete as 2008-2015. Missing data for variables collected in 2006-2007 ranged from $n=0$ to $n=139$ for 1017 registered trials, whereas for 2009-2012, the maximum number of data points missing for a variable was six. There were no missing data for trials registered between 2013 and 2015.

Objective 1: Proportion of prospective versus retrospective trial registrations 2006-2015, impact of introducing label in January 2013

From 2006-2015, the number of trials registered on the ANZCTR per year has been increasing steadily, and an increasing proportion of these trials were registered prospectively (figure 1). While in 2006 the number of trials registered on the ANZCTR was 446, of which 216 $(48 \%)$ were registered prospectively, in 2015 the number of trials registered on the ANZCTR was 1240, of which $754(61 \%)$ were registered prospectively. This increase in prospective registration has been particularly strong between $2006(48 \%)$ and $2012(63 \%)$ and has somewhat plateaued between 2012 and 2015 at around 64\%. After introduction of the prospective/retrospective registration label in January 2013, small increases were seen in prospective registration rates across 2013 (64\%) and 2014

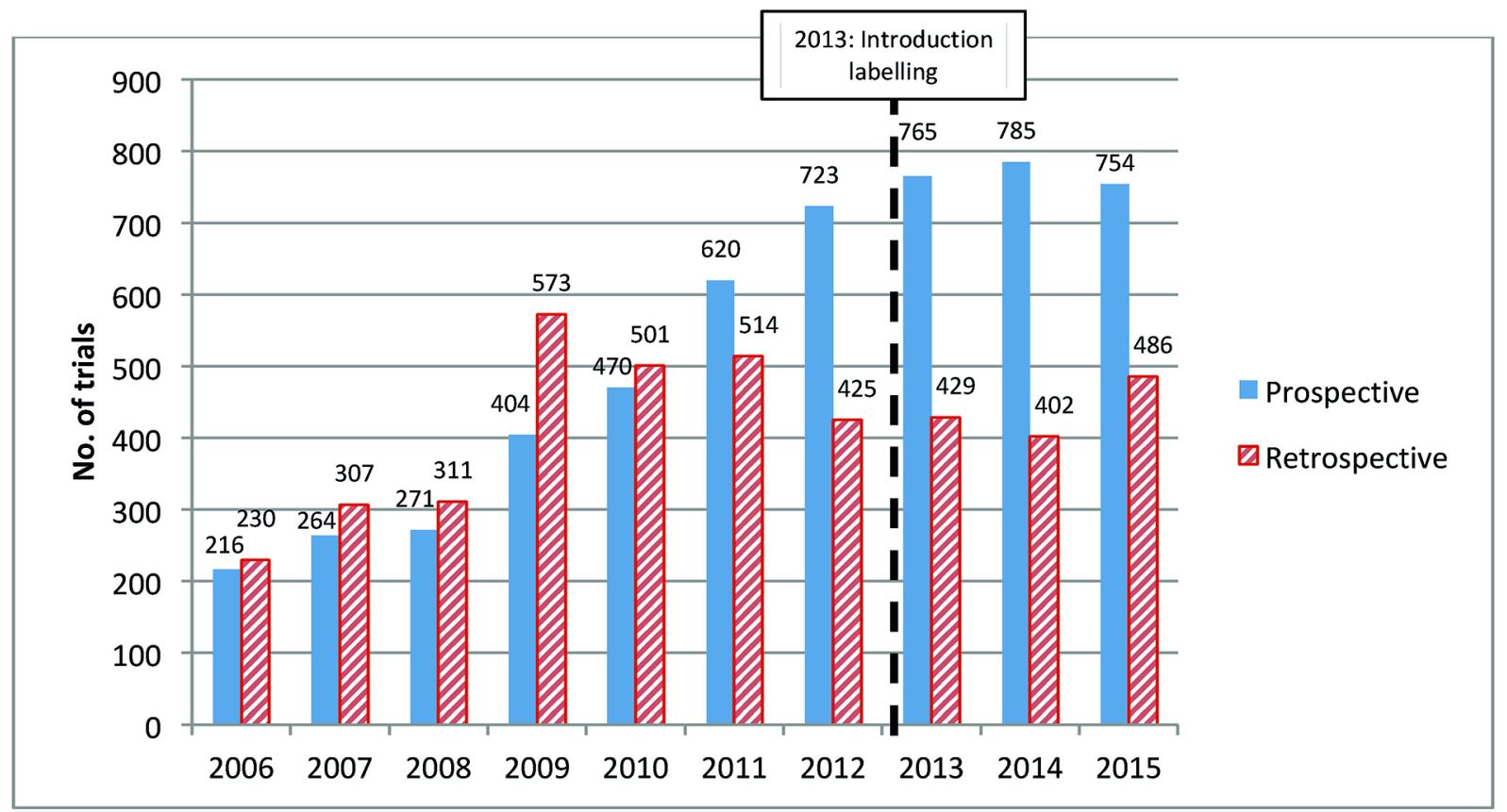

Figure 1 Absolute numbers of prospective and retrospective registrations on the ANZCTR by year. ANZCTR, Australian New Zealand Clinical Trials Registry. 


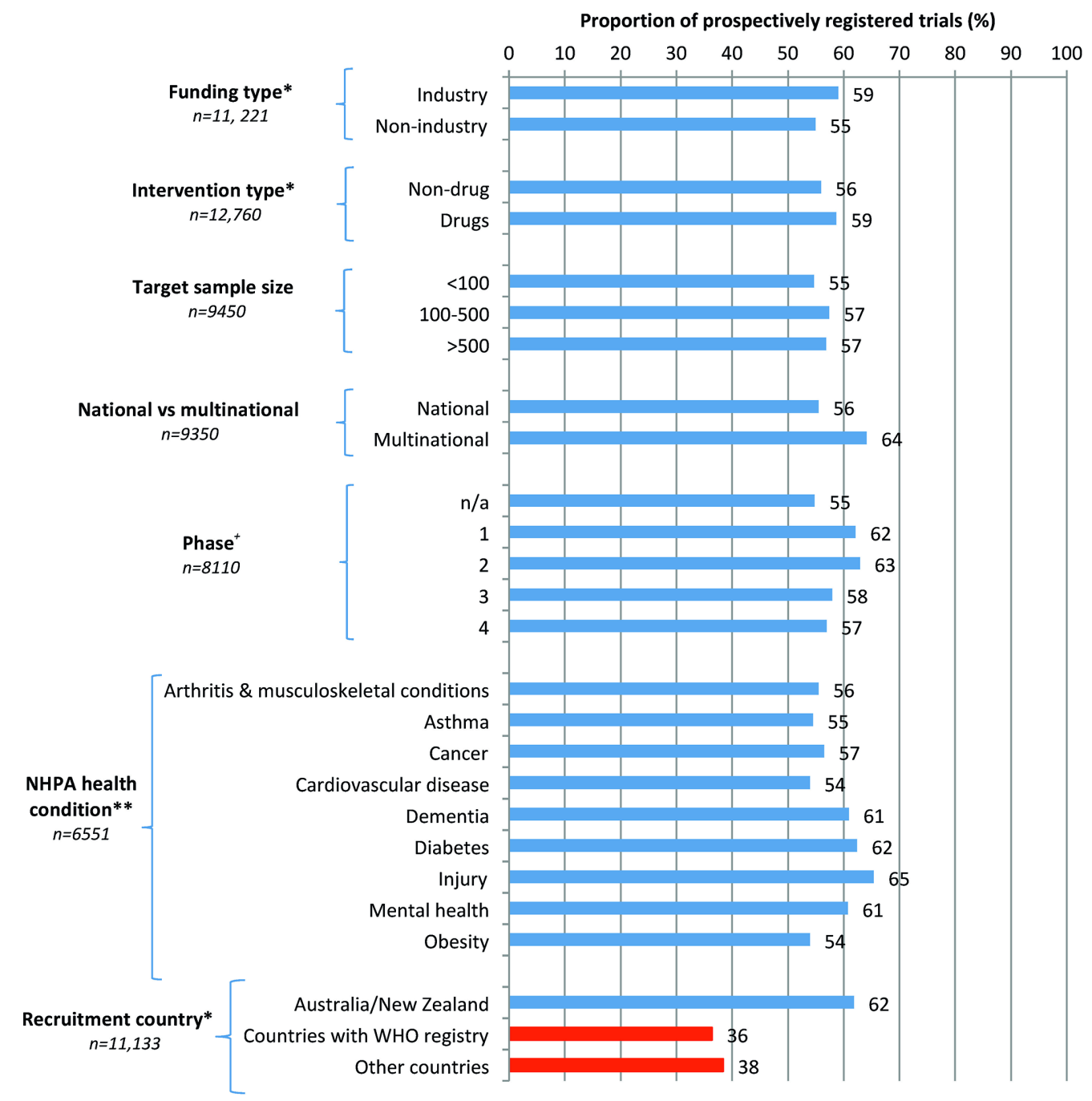

Figure 2 Prospective registration rates across various study characteristics. *A trial can add $>1$ entry for these fields. Therefore, the total number of data points is greater than the total number of trials. ${ }^{+}$Phase is an optional field on the ANZCTR registration form. ${ }^{* *}$ By Australian NHPA, which covers 6551/14682 (45\%) conditions in included trials. Note that a single trial can select up to three conditions. Proportion of missing values for mandatory fields was small. There were no missing values for health condition and target sample size, $2(0.02 \%)$ for funding type and $55(0.43 \%)$ for intervention type. There were 100 missing values for recruitment country $(0.90 \%)$ and multinational versus national (1.07\%). Almost all missing values are from 2006 to 2007 where data collection procedures were less stringent. ANZCTR, Australian New Zealand Clinical Trials Registry; NHPA, National Health Priority Area.

(66\%). However, compliance fell slightly in $2015(61 \%)$ (figure 1).

Objective 2: Prospective registration compliance on the ANZCTR by key study characteristics and country

As shown in figure 2, prospective registration compliance was relatively consistent across study characteristics. There were only very small differences in the proportion of prospectively registered trials by funding source, type of administered treatment, type of illness studied (classified by National Health Priority Areas), study phase, primary sponsor type and target sample size. However, larger differences were seen for study country. There were higher rates of prospective registration compliance among studies that were funded, registered, sponsored and that conducted their recruitment in Australia or New
Zealand, compared with those from other countries with a WHO Registry and countries without a WHO Registry.

A post hoc analysis was conducted to examine whether these differences between countries were due to different processing times arising from the prioritised handling of trials from Australia and New Zealand by ANZCTR staff. For this post hoc analysis, a more lenient categorisation of prospective registration was used, whereby submission (rather than final approval) of a registration form prior to study start was considered sufficient. In contrast to registration approval date, registration submission date is not influenced by different processing times for trials from different countries. While with this more lenient definition, the rate of prospectively registered studies in 2015 was $73 \%$ compared with $61 \%$ with the standard 


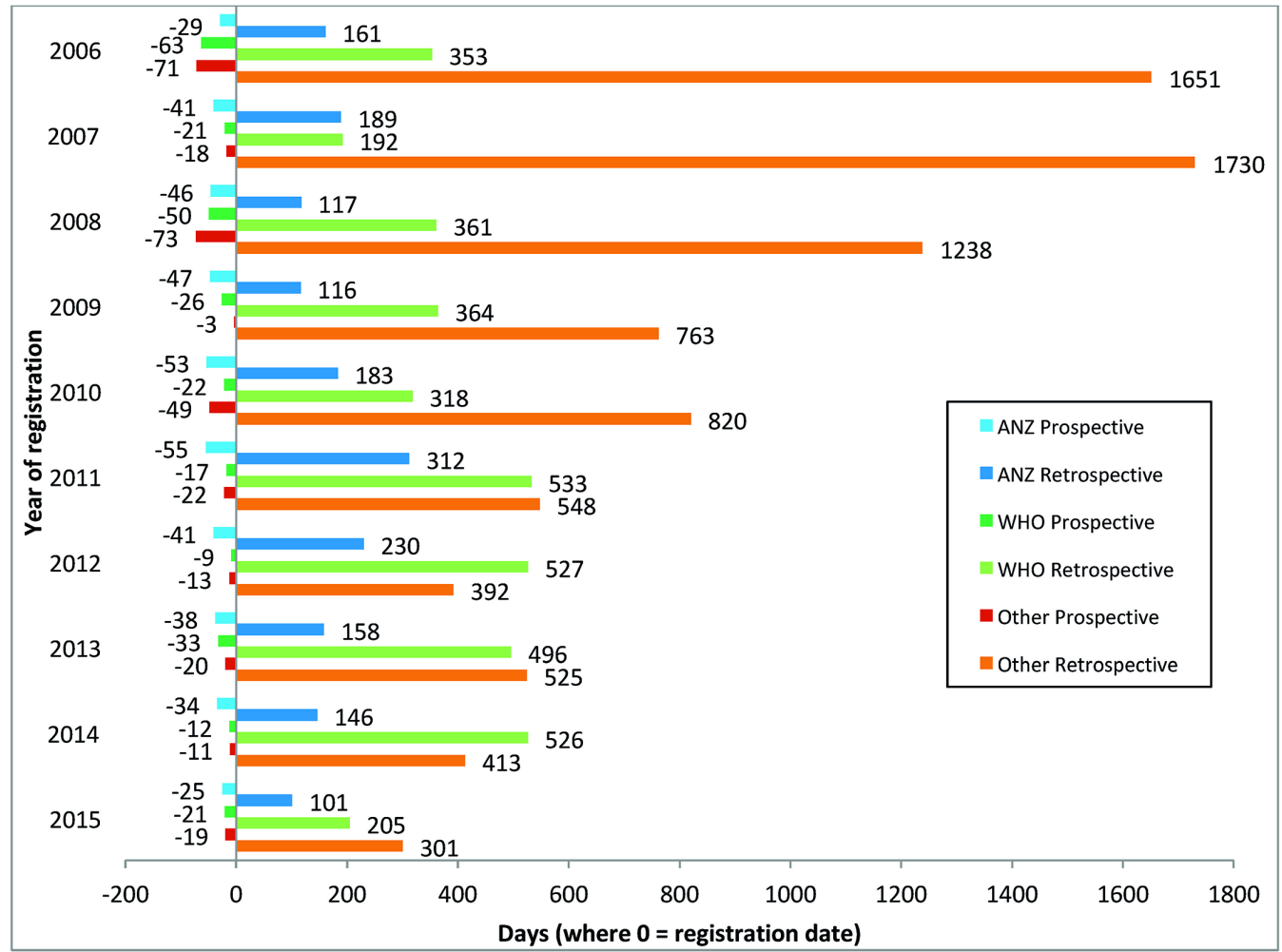

Figure 3 Median time (days) from registration to trial start by registrant country. ANZ, Australia/New Zealand; Other, all other countries; WHO, other countries with a WHO Network Registry. Missing: $n=4$.

definition, the increase in per cent points was consistent across country groups, and thus the difference in compliance between countries did not change (see online supplementary appendix 4).

Objective 3: Median time from registration on the ANZCTR to trial start date by year and country

The median time from registration approval to trial start was 36 days (IQR=90 days) for prospectively registered studies and 222 days (IQR=717 days) from trial start to registration approval for retrospectively registered studies. There was no clear trend over time from 2006 to 2015 for trials from Australia/New Zealand or countries with a WHO Registry (figure 3). However, a decline in median time from registration to trial start is apparent for trials from countries without a registry. Registrants from Australia and New Zealand registered their studies earliest in relation to trial start date, followed by countries with a WHO Registry and then other countries. For prospectively registered studies, the median approval date was 39 days ( $\mathrm{IQR}=97$ days) before trial start for studies from Australia and New Zealand, 21 days ( $\mathrm{IQR}=38$ days) for studies from countries with a WHO Registry and 19 days $(\mathrm{IQR}=40$ days $)$ for other countries. For retrospectively registered studies, the median time between trial start and registration approval was lowest for Australia (153 days, $\mathrm{IQR}=610$ days), followed by countries with a WHO Registry (median=409 days, IQR=817 days), followed by other countries (median=502 days, IQR=1058 days). Analyses were repeated for funding source country and primary sponsor country; the median times were comparable to the results for registrant country (see online supplementary appendix 5A,B).

\section{Part 2: Online registrant survey}

Survey invitations were sent to 404 registrants that were drawn in a stratified random sample of $15 \%$ of all retrospective registrations of interventional studies between 2010 and 2015 (figure 4). Fifty-one of these e-mails bounced, and of these, 34 were forwarded on to e-mail addresses retrieved in auto-reply, on the registration record or available elsewhere on the internet. A total of 149 responses were received, and 73 (49\%) of these chose to remain anonymous, while the remaining 76 $(51 \%)$ chose to provide their name and/or registration ID. This enabled us to extract key characteristics (see online supplementary appendix 6 ), which indicated that respondents were relatively representative of the eligible dataset. Ninety of the 149 respondents $(62 \%)$ identified themselves as the principal investigator/chief investigator of the registered study, while $30 \%$ were trial coordinator/ research staff (figure 5). Refer to online supplementary appendix 7 to see how survey responses were coded.

Objective 4: Reasons for non-compliance with the prospective registration requirement

Of the 148 respondents to this question, the most common reason cited for failure to register prospectively was lack of awareness (56\%) (figure 6). This included lack of awareness of the prospective registration requirement 


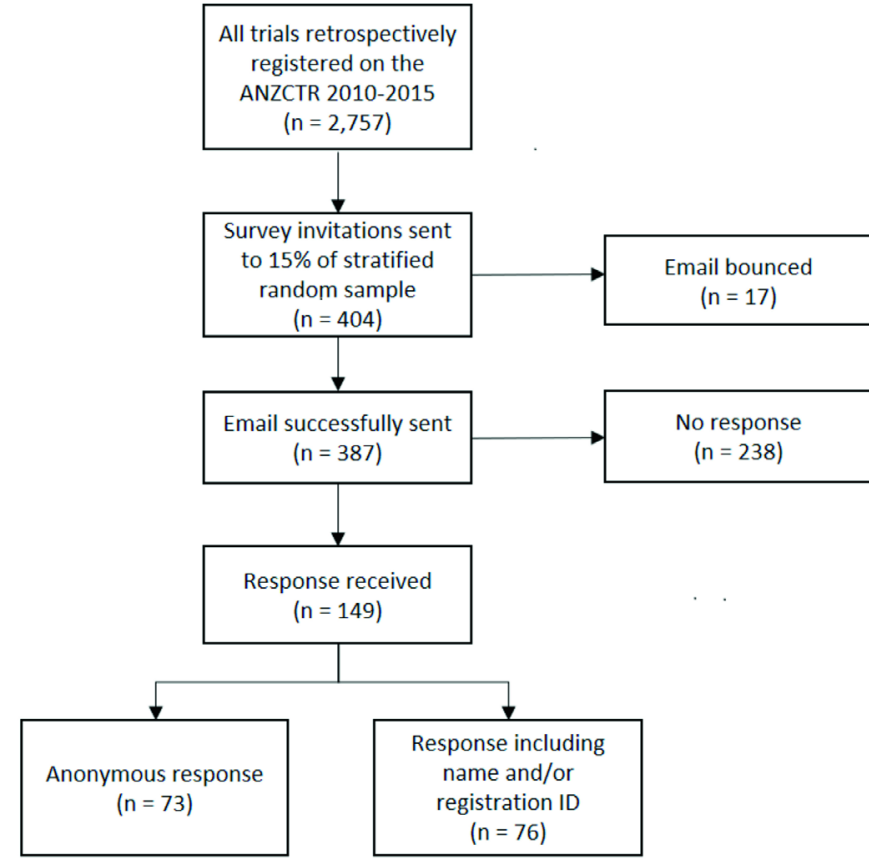

Figure 4 Survey flow chart. ANZCTR, Australian New Zealand Clinical Trials Registry.

in general, lack of awareness of the types of trials that need to be registered and not being aware of the importance of prospective registration. Lack of organisation was also common (28\%), with respondents citing lack of time, forgetfulness and confusion about who was responsible for registering. There was also uncertainty about what qualified as prospective registration; $9 \%$ of respondents did not realise final approval of their registration was still pending, while $8 \%$ thought that prospective submission was sufficient. Three per cent of respondents stated in open-ended responses that their respective study was a follow-up study or substudy, and part of their data thus already existed before their study was planned and registered.
Objective 5: Perceived usefulness of various proposed mechanisms to improve prospective registration compliance

Responses to this survey question are summarised in figure 7. Among the 140 respondents to this question, there was high agreement with suggesting incorporation of trial registration in the ethics approval procedures, with $74 \%$ agreeing it would be useful to have registration ID required on ethics form, to have ethics submission automatically uploaded to ANZCTR and/or have prospective registration on a checklist provided by ethics committees or other regulatory bodies. A lower proportion would find inclusion of the registration requirement in their organisation's procedures manual (21\% agreement) or software prompts (9\% agreement) useful.

Mechanisms suggested in open-ended responses related to processing time and clearer criteria. Two respondents asked for better notification of time frames for approval, while three thought that processing time was too lengthy and involved too many reviews and resubmissions and one asked for faster processing around holiday periods. Four respondents thought that there needed to be clearer criteria, for example around what types of studies require registration, and what constitutes prospective registration. Four respondents suggested other mechanisms, such as making release of funding contingent on trial registration.

\section{DISCUSSION}

Compliance with prospective registration of interventional studies on ANZCTR has plateaued at approximately $60 \%$ from 2012 to 2015, and this remains consistent regardless of sponsor or funder type, intervention type, health condition studied, size of trial and study phase. Studies recruiting in Australia/New Zealand were much more likely to be registered prospectively on ANZCTR (62\%) than those recruiting from other countries with a WHO Registry (36\%) or those recruiting from countries without a WHO Registry (38\%). In a survey

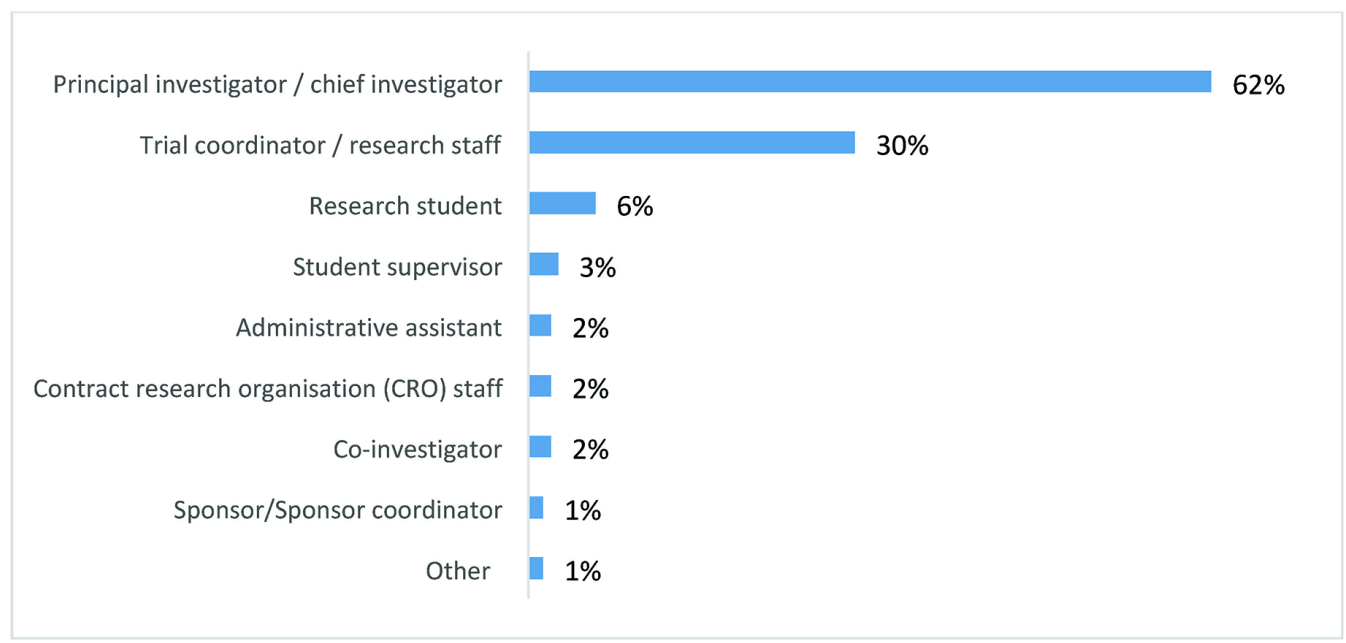

Figure 5 Role of person who registered trial. $n=149$; missing $n=0$. 


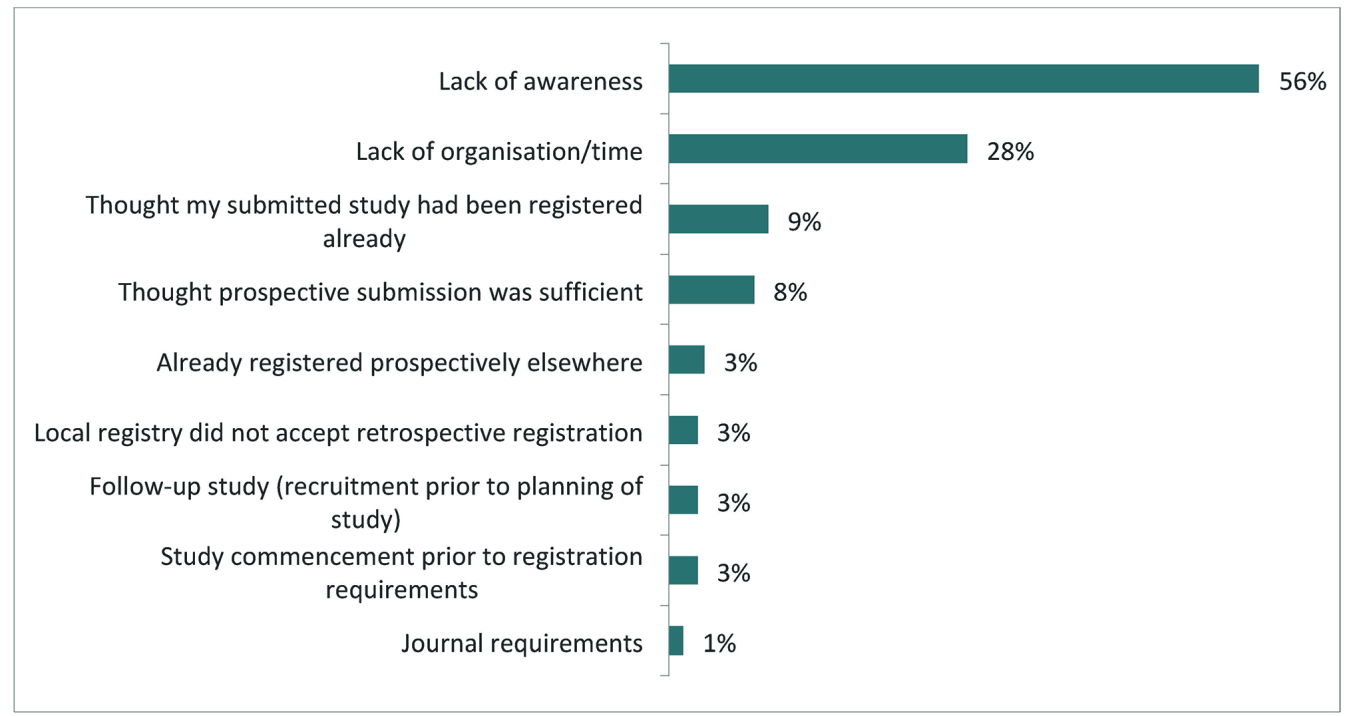

Figure 6 Reasons for not registering study prospectively. $n=148$, missing: $n=1$. Categorical survey responses were combined into broader categories for presentation of results. Where possible, free text responses were manually coded into the appropriate category; for responses that did not fit into an existing category new categories were created (this was the case for the bottom three categories).

of registrants who had retrospectively registered their study on ANZCTR, lack of awareness $(56 \%)$ and lack of organisation/time $(28 \%)$ were the two most common reasons cited for failure to register prospectively. About three quarters of respondents (74\%) agreed that linking registration to ethics approval would be useful to facilitate prospective registration compliance.

\section{Strengths and weaknesses}

To describe trends in prospective registration, this study used a complete dataset of 9450 interventional trials registered on ANZCTR from 2006 to 2015. This dataset included various key metrics of interest, most of which are mandatory on ANZCTR in accordance with WHO Trial Registration Minimum Dataset for Primary Registries. ${ }^{15}$
Response rates to the part 2 survey were higher than anticipated $(37 \%)$, and respondent characteristics fairly closely matched the full sample and eligible dataset, indicating that the stratified random sampling method used was effective (see online supplementary appendix 6). Moreover, to our knowledge this is the first survey of its kind to obtain feedback directly from registrants about reasons for retrospective registration and mechanisms to address this. A limitation is that our sample was restricted to the trials that were registered. Therefore, overall compliance with prospective registration would be lower, taking into consideration those studies that were never registered.

The approximately $60 \%$ compliance rate for prospective registration on ANZCTR from 2012 to 2015 is

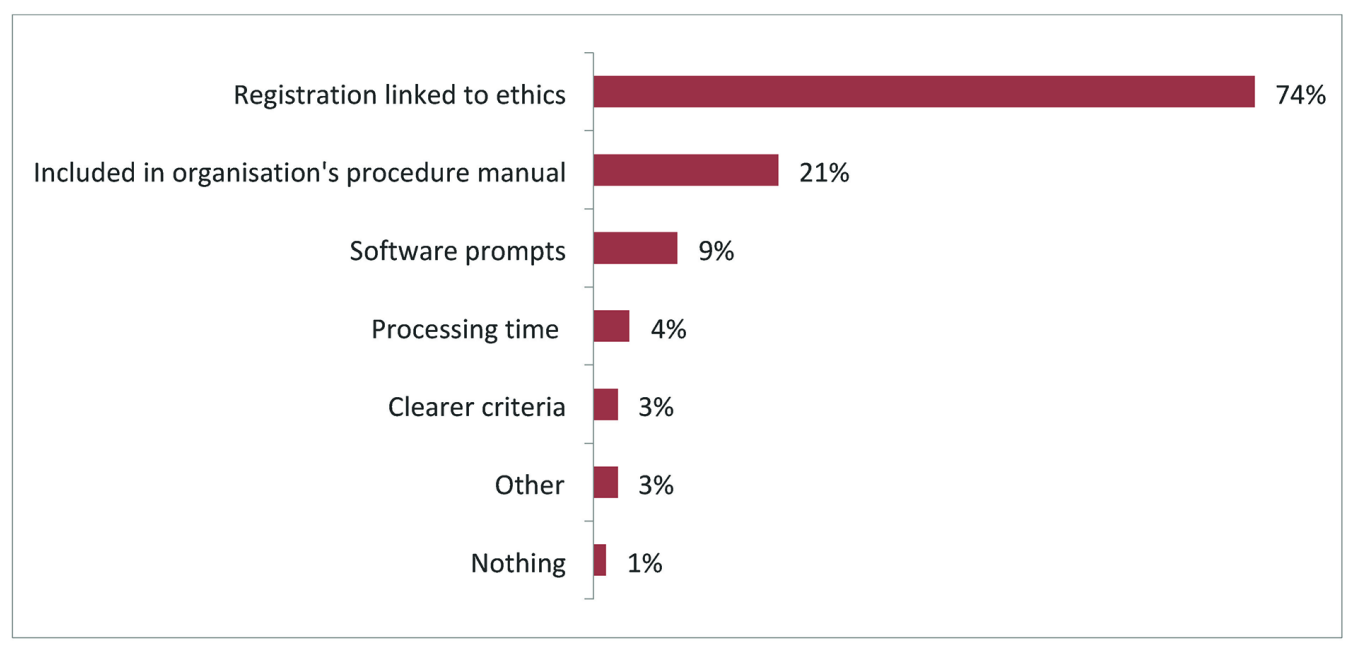

Figure 7 Factors that would have helped prevent retrospective registration on ANZCTR. $n=140$, missing: $n=9$. Categorical survey responses were combined into broader categories for presentation of results. Where possible, free text responses were manually coded into the appropriate category; for responses that did not fit into an existing category new categories were created (this was the case for the bottom four categories). ANZCTR, Australian New Zealand Clinical Trials Registry. 
higher than the approximately $50 \%$ rate reported across a random sample of all trials that were registered as interventional in 2012-2013 sourced from the WHO's ICTRP. ${ }^{16}$ However, it is comparable to ClinicalTrials.gov, where $67 \%$ of all interventional studies from 2012 to 2014 were reported to be registered 'on time', using a more conservative definition of registration before or within 3 months after the trial start date. ${ }^{17}$ This is comparable to our post hoc analysis, in which we found that $73 \%$ of trials were registered prospectively in 2015 when using the more lenient definition as submission (instead of approval) of registration prior to recruitment of the first participant. Analyses of the registration status of published journal articles across all intervention types have reported varying rates of prospective registration ranging from $24 \%$ to $72 \%,{ }^{11} 161819$ with the highest levels achieved by high-impact ICMJE-member journals, as might be expected since prospective registration is a requirement for submission to these journals. In fact, it is surprising that $28 \%$ of trials published in these journals are still retrospectively registered ${ }^{11}$ despite this requirement, indicating that journal editors intervene too late in the research process to be effective gatekeepers in preventing retrospective registration. In contrast to the present ANZCTR study, the study assessing trials registered at ClinicalTrials.gov found that industry-funded trials were more likely to be registered on time when compared with non-industry-funded trials. ${ }^{17}$

The survey offered several response options related to ethics approval, which may partly explain the high rate of agreement with ethics-related mechanisms to improve prospective registration compliance. However, these options reflect the experience from pilot surveys and communication with registrants and experts in the field. Moreover, respondents were given the option to add other reasons in an open-text field, and additional recommendations about incorporating registration into ethics approval was the most frequent reply in this section.

\section{Interpretation and implications: incorporating registration into ethics approval}

To maximise research transparency and minimise bias, clinical trials need to be registered prior to enrolment of the first participant, that is, prospectively. This study shows that compliance with prospective registration remains suboptimal, and action needs to be taken to address this. To be most effective, global measures such as the Declaration of Helsinki and ICMJE statement need to be complemented with implementation and enforcement of local measures to improve prospective registration compliance. ${ }^{20}$ Local measures may include legislation, and enforcement by funders, ethic committees and institutions and the availability of registries in the local language. ${ }^{20}$

Incorporation of registration into the ethics approval process is one possible mechanism which appears to have a high level of support among researchers based on the present survey results. Possible ways in which ethics approval could be linked to registration include requiring a registration ID on the ethics application form, having prospective registration on a checklist provided by ethics committees and having the relevant data from an ethics submission electronically uploaded to a trial registry. Making registration mandatory for all types of trials via ethics committees may complement legislation in this area: in countries with legislation on trial registration, the law tends not to cover all types of clinical trials, for example, in the USA, only certain clinical trials of drugs, biologics and devices that are subject to Food and Drug Administration regulation are required to be registered by legislation. ${ }^{21}$

In Australia and many other countries, trials require ethics approval prior to commencement. In 2014, the Australian Health Ethics Committee agreed that prospective trial registration should be a mandatory condition of ethics approval (Simes 2015, personal communication). If all ethics committees added such a clause to their standard approval letters, any trials proceeding without prospectively registering would be in breach of their ethics approval. Furthermore, this would increase awareness and highlight the importance of prospective registration. The UK's Health Research Authority (HRA) has already recognised and acted on the opportunity to incorporate trial registration into the ethics approval process. In September 2013, they introduced a new rule requiring trial registration as a formal condition of Research Ethics Committee (REC) approval. ${ }^{22}$ Two years later, they conducted an audit which found that of 794 trials with a favourable ethical opinion during the reporting period, only $434(55 \%)$ could be found on a publicly accessible database and $49(6 \%)$ were phase I trials which were allowed to defer registration due to commercial sensitivities. ${ }^{23}$ They noted that this does not necessarily mean that the outstanding trials have not been registered but could perhaps be due to limitations in their search strategy and the allowance of a 6-week leeway for registration after recruitment of the first UK participant. They planned to e-mail sponsors to confirm actual registration status and to further elucidate reasons for non-registration. Those who are in breach of the requirement are managed with REC standard operating procedures-legal sanctions do not apply. ${ }^{24}$ The UK experience highlights some complexities that may arise in implementing registration into ethics approval.

To minimise bureaucratic burden for researchers and facilitate compliance, it would be useful to electronically link the ethics form to the registration interface. Currently, in Australia, investigators wishing to undertake a clinical trial need to submit an ethics application form to one or more Human Research Ethics Committees (HRECs), enter data regarding key aspects of their trial on a recognised trial registry and where applicable submit a Clinical Trial Notification/Clinical Trial Exemption (CTN/CTX) form to the Therapeutic Goods Administration (TGA). Data entry for these three agencies is not currently harmonised nor are data exchanged. Data lodged with the TGA and HRECs is not publicly available. 
We propose the development of information technology infrastructure that will enable researchers to seamlessly register their trials prospectively as part of the ethics approval process, thereby ensuring all trials being conducted in Australia are included in the national ANZCTR database. A similar solution has been proposed by Chalmers ${ }^{25}$ in the UK context using the HRA's integrated research application system (www.myresearchproject.org.uk).

Future research could assess the effect of requiring registration as a condition of ethics approval among a sample of ethics committees. Initially, the focus could be on feasibility of implementation and analysis of barriers and facilitators, including feedback from the ethics committees and researchers. Subsequent work could focus on the effectiveness of implementation in terms of change in prospective registration rates.

\section{CONCLUSION}

Prospective clinical trial registration is an essential tool to maximise research transparency and improve health outcomes. Further efforts are needed to increase compliance rates, which have plateaued at about $64 \%$ on ANZCTR since 2012. Incorporating registration into the ethics approval process is one potential solution with strong support from researchers. This would also address the most commonly cited barrier to prospective registration compliance, a lack of awareness, as Australian interventional trials require ethics approval prior to commencement.

Acknowledgements The authors would like to thank ANZCTR IT officers, Thuyen Vu and Ryan Sausa, for extracting the required data from the ANZCTR database. We also acknowledge the facilities and the scientific and technical assistance of the ANZCTR at the NHMRC Clinical Trials Centre, University of Sydney. ANZCTR is supported by funding from the National Health and Medical Research Council (NHMRC) and Therapeutic Innovation Australia (TIA). TIA is supported by the Australian Government through the National Collaborative Research Infrastructure Strategy (NCRIS) programme.

Contributors KEH and LMA participated in development of the study protocol. KEH and ALS performed all data analysis. KEH and ALS drafted the manuscript. All authors read and approved the final version of the manuscript.

Funding This research received no specific grant from any funding agency in the public, commercial or not-for-profit sectors.

Competing interests All authors are staff at the ANZCTR.

Patient consent Not required.

Ethics approval University of Sydney Human Research Ethics Committee.

Provenance and peer review Not commissioned; externally peer reviewed.

Data sharing statement $A$ full dataset of results is available from the corresponding author upon receiving a reasonable request.

Open Access This is an Open Access article distributed in accordance with the Creative Commons Attribution Non Commercial (CC BY-NC 4.0) license, which permits others to distribute, remix, adapt, build upon this work non-commercially, and license their derivative works on different terms, provided the original work is properly cited and the use is non-commercial. See: http://creativecommons.org/ licenses/by-nc/4.0/

(c) Article author(s) (or their employer(s) unless otherwise stated in the text of the article) 2018. All rights reserved. No commercial use is permitted unless otherwise expressly granted.

\section{REFERENCES}

1. Dickersin K, Rennie D. Registering clinical trials. JAMA 2003;290:516-23.

2. Dickersin K. Why register clinical trials? Control Clin Trials 1992;13:170-7.

3. Chalmers I, Dickersin K, Chalmers TC. Getting to grips with Archie Cochrane's agenda. BMJ 1992;305:786-8.

4. Simes RJ. Publication bias: the case for an international registry of clinical trials. J Clin Oncol 1986;4:1529-41.

5. Easterbrook P. Reducing publication bias. Br Med J 1987;295:1347.

6. Weber WE, Merino JG, Loder E. Trial registration 10 years on. BMJ 2015;351:h3572.

7. Laine $\mathrm{C}$, Horton $\mathrm{R}$, DeAngelis $\mathrm{CD}$, et al. Clinical trial registration: looking back and moving ahead. Lancet 2007;369:1909-11.

8. De Angelis C, Drazen JM, Frizelle FA, et al. Clinical trial registration: a statement from the International Committee of Medical Journal Editors. Lancet 2004;364:911-2.

9. World Medical Assocation. Declaration of Helsinki: Ethical principles for medical research involving human subjects, 2008.

10. World Health Organisation. International Clinical Trials Registry Platform (ICTRP), 2011. http://www.who.int/ictrp/trial_reg/en/index. html.

11. Dal-Ré R, Ross JS, Marušić A. Compliance with prospective trial registration guidance remained low in high-impact journals and has implications for primary end point reporting. J Clin Epidemiol 2016;75:100-7.

12. World Health Organization. International standards for clinical trial registries. 2012 http://apps.who.int/iris/bitstream/10665/76705/1/ 9789241504294_eng.pdf?ua=1 (accessed 11 Sept 2017).

13. Australian Institute of Health and Welfare and Commonwealth Department of Health and Family Services. First report on National Health Priority Areas 1996, 1997.

14. R Core Team. R: A language and environment for statistical computing, 2013.

15. World Health Organization International Clinical Trials Registry Platform. WHO Trial Registration Data Set (Version 1.2.1). http:// www.who.int/ictrp/network/trds/en/ (accessed Sep 2017).

16. Viergever RF, Karam G, Reis A, et al. The quality of registration of clinical trials: still a problem. PLoS One 2014;9:e84727.

17. Zarin DA, Tse T, Williams RJ, et al. Update on trial registration 11 years after the ICMJE policy was established. N Engl J Med 2017;376:383-91.

18. Harriman SL, Patel J. When are clinical trials registered? An analysis of prospective versus retrospective registration. Trials 2016;17:187.

19. Dal-Ré R, Marušić A. Prevention of selective outcome reporting: let us start from the beginning. Eur J Clin Pharmacol 2016;72:1283-8.

20. Viergever RF, Li K. Trends in global clinical trial registration: an analysis of numbers of registered clinical trials in different parts of the world from 2004 to 2013. BMJ Open 2015:5:e008932.

21. U.S Food and Drug Administration. Food and Drug Administration Amendments Act (FDAAA) of 2007. https://www.fda.gov/ RegulatoryInformation/LawsEnforcedbyFDA/SignificantAmendments totheFDCAct/FoodandDrugAdministrationAmendmentsActof2007/ default.htm (accessed 12 Feb 2011).

22. Dyer C. UK clinical trials must be registered to win ethics committee approval. BMJ 2013;347:f5614.

23. Health Research Authority. Clinical Trial Registration Audit Report. 2015 http://www.hra.nhs.uk/wp-content/uploads/2015/2007/20092015-2014lii-Clinical-Trial-Registration-Audit-Report_V2011-2013. docx (accessed 17 Aug 2017).

24. Health Research Authority. HRA and Research Transparency: Clinica trial registration. 2015 http://www.hra.nhs.uk/documents/2015/200 8/qa_july2015_hraandresearchtransparency-september-2015.pdf (accessed 17 Aug 2017).

25. Chalmers I. Health Research Authority's great leap forward on UK trial registration. BMJ 2013;347:f5776. 